\title{
Characterization of Point Mutations in the Same Arginine Codon in Three Unrelated Patients with Ornithine Transcarbamylase Deficiency
}

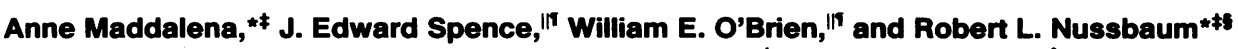 \\ ${ }^{*}$ Howard Hughes Medical Institute and the Departments of ${ }^{\ddagger}$ Human Genetics and ${ }^{\S}$ Pediatrics, University of Pennsylvania School \\ of Medicine, Philadelphia, Pennsylvania 19104; and "Howard Hughes Medical Institute and the 'Institute of Molecular Genetics, \\ Baylor College of Medicine, Houston, Texas 77030
}

\begin{abstract}
Point mutations in the $\mathrm{X}$-linked ornithine transcarbamylase (OTC) gene have been detected at the same Taq $I$ restriction site in 3 of 24 unrelated probands with OTC deficiency. A de novo mutation could be traced in all three families to an individual in a prior generation, confirming independent recurrence. The DNA sequence in the region of the altered Taq I site was determined in the three probands. In two unrelated male probands with neonatal onset of severe OTC deficiency, a guanine $(G)$ to adenine $(A)$ mutation on the sense strand (antisense cytosine $[\mathrm{C}]$ to thymine $[\mathrm{T}]$ ) was found, resulting in glutamine for arginine at amino acid 109 of the mature polypeptide. In the third case, where the proband was a symptomatic female, $\mathrm{C}$ to $T$ (sense strand) transition converted residue 109 to a premature stop. These results support the observation that Taq I restriction sites, which contain an internal CG, are particularly susceptible to $\mathbf{C}$ to $\mathbf{T}$ transition mutation due to deamination of a methylated $C$ in either the sense or antisense strand. The OTC gene seems especially sensitive to $C$ to $T$ transition mutation at arginine codon 109 because either a nonsense mutation or an extremely deleterious missense mutation will result.
\end{abstract}

\section{Introduction}

Ornithine transcarbamylase (OTC) ${ }^{1}$ (E.C. 2.1.3.3) is the Xlinked hepatic enzyme that catalyzes the intra-mitochondrial condensation of ornithine and carbamylphosphate in the second step of the urea cycle. Males with classical OTC deficiency (McKusick 31125) experience neonatal ammonia intoxication with severe or fatal neurological damage (1). Female carriers may be asymptomatic, but often experience some degree of protein intolerance. They, as well as males with partial enzymatic deficiencies, may have a normal lifespan but are at risk

This work was presented in part at the American Society of Human Genetics, Philadelphia, PA, 2-5 November 1986.

Dr. Maddalena's present address is Genetics and IVF Institute, Fairfax, VA. Dr. Spence's present address is Medical College of Virginia, Richmond, VA.

Address reprint requests to Dr. Robert L. Nussbaum, Howard Hughes Medical Institute, Department of Human Genetics, University of Pennsylvania School of Medicine, 37th and Hamilton Walk, Philadelphia, PA 19104-6072. 1988.

Received for publication 4 March 1988 and in revised form 1 June

1. Abbreviations used in this paper: A, adenine; $\mathrm{C}$, cytosine; $\mathrm{G}$, guanine; OTC, ornithine transcarbamylase; RFLP, restriction fragment length polymorphism; $T$, thymine.

J. Clin. Invest.

(c) The American Society for Clinical Investigation, Inc.

$0021-9738 / 88 / 10 / 1353 / 06 \quad \$ 2.00$

Volume 82, October 1988, 1353-1358 for stress-induced hyperammonemic crises and incremental neurological damage (1).

OTC activity is usually undetectable in liver tissue from severely deficient males, although OTC-specific mRNA and/ or the protein product may be present $(2,3)$. Males with partial OTC activity may have diminished enzyme levels, or normalto-high levels with abnormal kinetic properties (2). In DNA studies, a few cases of severe deficiency have been shown to be caused by deletion of part or all of the OTC gene (4-7), and we have seen one male with a mild deficiency due to mosaicism for a deletion within this gene (Maddalena, A., and R. L. Nussbaum, manuscript in preparation). Biochemical studies $(2,3)$ and mouse models $(8,9)$ suggest that a variety of different point mutations may be responsible for heterogeneity in OTC deficiency. As a consequence, family studies are usually accomplished with restriction fragment length polymorphisms (RFLP) rather than by direct detection of the mutation (4, 10-12; Nussbaum, R. L., unpublished observations).

The detection of a point mutation by a restriction enzyme depends on the unlikely coincidence that the mutation creates a new recognition site or eliminates an existing one. However, restriction sites containing the sequence cytosine (C):guanine (G) appear to have an intrinsically higher mutation risk (13). The relatively rare CG dinucleotide is the major site of methylation in human DNA; once methylated, the $C$ in either strand may be mutated to a thymidine by spontaneous deamination. Accordingly, we routinely screen DNA from OTC patients using the enzyme Taq I (recognition sequence TCGA), in addition to using Msp I (CCGG) for RFLP studies. Abnormal Taq I restriction fragments have been detected in 4 of 24 unrelated families analyzed. In three of these unrelated families, an alteration of the same Taq I site was seen, resulting in the loss of the usual Taq I fragment of $1.8 \mathrm{~kb}$ and the appearance of a new 3.4-kb fragment. The fact that these mutations occurred independently in three families suggested that this particular Taq I site in the OTC gene was prone to mutation. We have now localized this Taq I site within the OTC gene, determined its DNA sequence, and identified two different $\mathrm{C}: \mathrm{G}$ to thymine (T):adenine (A) transition mutations responsible for the severe OTC deficiency in these three families.

\section{Methods}

OTC cDNA probes. pHOC1 (14) contains a 1.2-kb OTC cDNA, which is lacking $\sim 0.4 \mathrm{~kb}$ of $5^{\prime}$ exonic sequence. pHOCl detects genomic Taq I restriction fragments of 4.6, 3.8, 2.7, 1.9 (faint), 1.8, and $1.4 \mathrm{~kb}$.

pHOC 3 contains a full-length OTC $1.5-\mathrm{kb}$ cDNA insert originally isolated from a human liver cDNA $\lambda$ gt 11 library by hybridization with pHOCl (A. Maddalena and R. L. Nussbaum). The insert encodes functional OTC enzyme when inserted in an expression vector and transfected into cultured cells (Nussbaum, R. L., unpublished observations). Additional Taq I genomic fragments detected by $\mathrm{pHOC} 3$ are 0.9 and $0.8 \mathrm{~kb}$, and a second fragment of $4.6 \mathrm{~kb}$. 
Subfragments of OTC cDNA were prepared by digestion of pHOC1 and pHOC3 by appropriate restriction enzymes and separation of fragments on low melting point agarose.

Southern blots. DNA was prepared from nuclei of peripheral leukocytes or lymphoblastoid cells by SDS and proteinase K digestion, phenol extraction, and ethanol precipitation. Restriction enzymes were purchased from New England Biolabs (Beverly, MA) or Promega (Madison, WI) and used as directed by the manufacturer. Electrophoresis in $0.8 \%$ agarose and blotting in $10 \times$ standard saline citrate (SSC) followed standard protocols. Probes consisted of cDNA inserts or insert fragments isolated in low melting point agarose and labeled by random primer extension (15) to a specific activity of $10^{9} \mathrm{dpm} / \mu \mathrm{g}$. Hybridizations using over $10^{6} \mathrm{dpm} / \mathrm{ml}$ were incubated at $42^{\circ} \mathrm{C}$ for $24-48 \mathrm{~h}$ in solutions containing $50 \%$ formamide. Final washes were at $65^{\circ} \mathrm{C}$ in $2 \times \mathrm{SSC}, 0.1 \%$ SDS. Blots were exposed to Kodak XAR film for autoradiography.

Cloning and sequencing. A phage library of $2 \times 10^{6}$ clones was made from high molecular weight lymphoblastoid DNA from the male proband of family Bo (previously designated family A) (14). DNA was partially digested with Sau $3 \mathrm{~A}$ and cloned into the Bam HI sites of EMBL3 (Promega). Plaques were screened with cDNA probes. Phage DNA was prepared by polyethylene glycol precipitation and mapped by double restriction digestion. Subclones in pUC19 were also screened with cDNA probes. Sequencing was performed by the chain termination method with dideoxynucleotides in $\mathrm{M} 13 \mathrm{mp} 19$ subclones.

Oligonucleotide probes. Radiolabeled oligonucleotide probes of high specific activity were prepared by the primer extension method (16). A set of 18-base oligonucleotides was synthesized that was complementary to the genomic sequence surrounding the altered Taq I site, but differed from one another at position 9 or 10 . A complementary 8-base primer was annealed to the $3^{\prime}$ end of each of these 18-base oligomers and extended using Klenow fragment to incorporate $\left[{ }^{32} \mathrm{P}\right] \alpha$ dATP at three or four sites per strand. Each labeled probe was separated from its template by polyacrylamide/urea elecrophoresis. Differential migration of probe and template was accomplished by retention of the dimethoxytrityl group on each primer strand (16). After autoradiography, the probes were extracted from the gel by the crush-andsoak method.

Oligonucleotide hybridization. Oligonucleotide hybridization was performed on nitrocellulose blots. Hybridization and washing followed the tetramethylammonium chloride procedure (17) as described by DiLella and Woo (18), except that probe concentration was $10^{5}-10^{6}$ $\mathrm{cpm} / \mathrm{ml}$. A washing temperature of $54^{\circ} \mathrm{C}\left(3-4^{\circ}\right.$ below the dissociation temperature for an 18-mer) was used.

Case histories. Families Bo and En have been described (previously designated families A and B) (14). The probands in both were severely OTC-deficient male infants whose partially deficient mothers were diagnosed after the birth of the affected child. The proband of family Bo was originally reported as severely neurologically damaged; he died at the age of $2 \frac{1}{2} \mathrm{yr}$, having achieved no growth or developmental milestones since infancy. The proband in family En died during the first week of life. Southern blots of the OTC genes of both infants showed the absence of a normal 1.8-kb Taq I fragment and the appearance of an abnormal 3.4-kb fragment. Their mothers and other maternal relatives with demonstrable protein intolerance also had the $3.4-\mathrm{kb}$ marker. In both families, a generation was available for testing in which neither the 3.4-kb fragment nor protein intolerance were found.

The proband in family $\mathrm{Ma}$ is a 21 -yr-old female who had sporadic episodes of neurological dysfunction with symptoms including severe headaches, behavioral changes, and loss of consciousness. After an episode at age 12, she was diagnosed as partially OTC deficient on the basis of hyperammonemia $(257 \mathrm{~nm} /$ liter $)$ and orotic aciduria $(8,266$ $\mu \mathrm{g} / \mathrm{mg}$ creatinine) following a meal of $0.75 \mathrm{~g}$ protein $/ \mathrm{kg}$ body $\mathrm{wt}$. She presently avoids high protein foods voluntarily but is somewhat noncompliant in taking her prescribed sodium benzoate and calcium gluconate. Since diagnosis, she has required occasional hospitalization for mild to moderate hyperammonemia. She has completed high school but is recognized as intellectually slow. Partial OTC deficiency was also diagnosed by protein-loading studies in her mother, who has an aversion to proteins, and one sister, who is asymptomatic. Another sister and the maternal grandmother have normal protein tolerance. There are no male siblings, and no male neonatal deaths in the extended family.

\section{Results}

Southern blot analysis. Southern blots of the OTC gene in family Ma showed normal restriction patterns with every enzyme tested except Taq I (J. E. Spence and W. E. O'Brien). The three females with confirmed OTC deficiency had an aberrant fragment at $3.4 \mathrm{~kb}$, in addition to the normal Taq I fragments (Fig. 1). The same aberrant fragment at $3.4 \mathrm{~kb}$ was seen in the OTC heterozygotes and hemizygotes in the Bo and En families (14). The 3.4-kb marker appeared nowhere else in the family. As with families Bo and En, it is possible to pinpoint a generation in the Ma family in which the metabolic deficiency and the 3.4-kb marker jointly appeared: individual II.1 has both traits, while her mother (I.2) has neither. Barring low-level somatic or germline mosaicism, her father (I.1), who was not available for study, is excluded from having OTC deficiency by history, and from carrying the 3.4-kb marker by the normal Southern blot of a second daughter (data not shown).

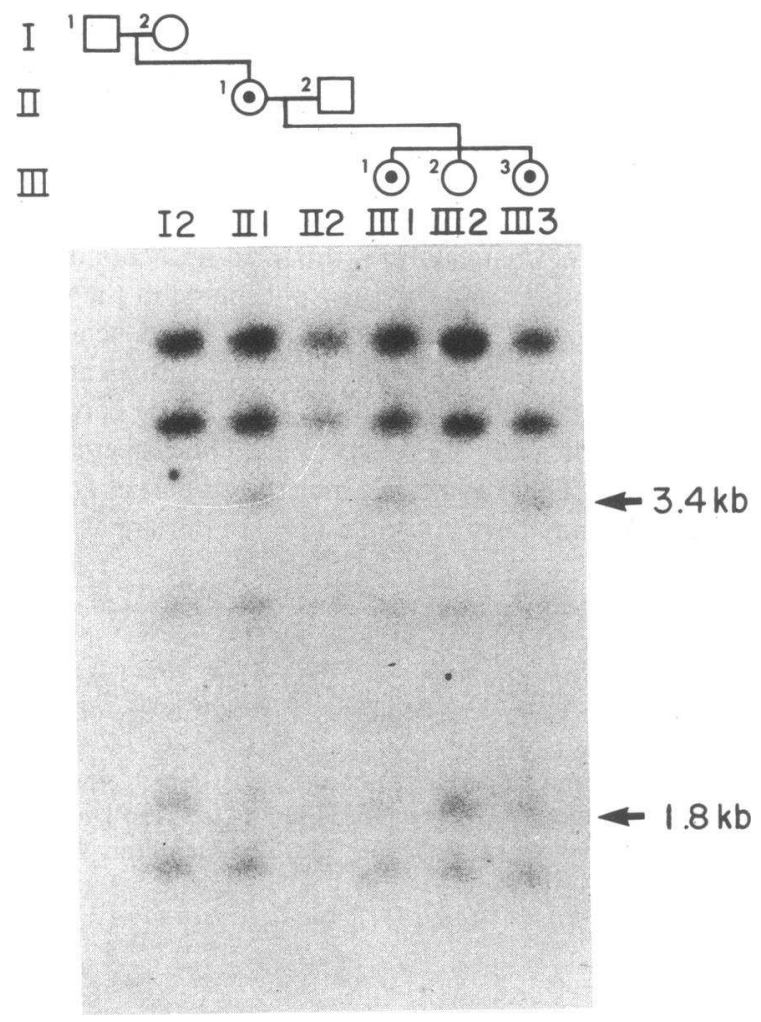

Figure 1. Pedigree and Taq I restriction analysis of family Ma, showing the coinheritance of the abnormal 3.4-kb fragment with OTC deficiency. Open squares, unaffected males; open circles, unaffected females; circles with dots, females with demonstrated OTC deficiency. The proband is individual Ma.III.3. Pedigree symbols are aligned over the lane containing that individual's sample. The radiolabeled probe was $\mathrm{pHOC} 3$. 
The loss of a restriction site between two fragments of 1.6 and $1.8 \mathrm{~kb}$ would create a fragment of $3.4 \mathrm{~kb}$. Because no 1.6-kb fragment was detected with even the full-length cDNA probe pHOC3, we postulated that if the altered Taq I site were in an exon, it must be located near an intron/exon boundary. Rather than attempt allele-specific hybridizations with oligonucleotide probes based solely on the cDNA sequence, we elected first to determine the genomic sequence in the critical region of the OTC gene of the male proband of family Bo.

Cloning of the OTC gene of proband $B o$. As a preliminary step in cloning, subfragments of the OTC cDNA were tested in order to identify a probe specific for the altered Taq I fragment. A fragment from the $5^{\prime}$ end of pHOC 1 , designated pHOC1-A, detects only the 3.4-kb genomic Taq I fragment in the DNA of the family Bo proband, or the $1.8-\mathrm{kb}$ fragment in normal DNA. pHOC1-A extends from the 5 end of $\mathrm{pHOC1}$ (approximately base 270) to the Pvu II site at base 479 (numbered from the leader peptide cleavage site as by Horwich et al. [19]), and contains a single Taq I site at base 324 in the cDNA. pHOC1-A, along with the full length cDNA probe, was used to screen $10^{6}$ plaques containing genomic DNA from proband Bo. Of two OTC-positive plaques found, one, $\lambda \mathrm{JB} 1$, was also positive with the specific probe pHOC1-A.

The 18-kb insert of $\lambda \mathrm{JB} 1$ was subcloned and partially sequenced as shown in Fig. 2. It contained sequences from the central to $3^{\prime}$ region of the OTC gene, as shown by hybridization with cDNA fragments (Maddalena, A., and R. L. Nussbaum, data not shown). The $\lambda \mathrm{JB} 1$ insert was subcloned into pUC19 as a series of Pst I fragments. The exon-containing Pst I subclones were identified, and their linear order was confirmed, by hybridization with OTC cDNA fragments. A plasmid containing the region of the mutation, pJB19, was detected with probe $\mathrm{pHOC1}-\mathrm{A}$. Its $1.7-\mathrm{kb}$ insert was subcloned into M13 and sequenced in both directions from an internal Rsa I site as indicated.

Genomic sequence of proband $\mathrm{Bo}$. The genomic sequence from proband Bo corresponded with two exceptions to a central portion of the published cDNA sequence, defining an exon of 154 bases (Fig. 2). Flanking the exon are introns with standard splice signals. Of the two alterations found in the sense sequence, one is a silent $T$ to $G$ transversion in the third position of the codon for $\mathrm{Leu}_{119}$. The other is a $\mathrm{G}$ to $\mathrm{A}$ transition that changes codon 109 from CGA (arginine) to CAA (glutamine). Together with the preceding $T$, the normal CGA codon constitutes a Taq I site, which a $G$ to A transition would destroy. In addition to changing the Southern blot pattern for Taq I, this mutation causes an amino acid substitution that could have a significant effect on protein stability or function. Also, the inability of cDNA probes to detect a normal genomic Taq I fragment of $1.6 \mathrm{~kb}$ in Southern blots of normal individuals is now easily explained by the fact that there are only 34 bases of exonic sequence $5^{\prime}$ to this Taq I site.

Detection of the mutations in families En and Ma with oligonucleotide probes. The genomic sequence was then used to produce a series of synthetic oligonucleotide probes for direct analysis of the mutation site in families En and Ma. The normal, or arginine specific, probe had the sequence TATTGGCTCGAGTGTATA, with the Taq I site centrally located (underlined). The probe corresponding to the glutamine missense mutation contained $A$ instead of $G$ at position 10. A third probe had the other likely substitution, a $C$ to $T$ transition at position 9 , which in this reading frame would create a stop (UGA) mutation.

This set of probes was hybridized to Southern blots of Pst I digested DNA and washed under conditions requiring a perfect match $(18,19)$. Specific hybridization was expected to
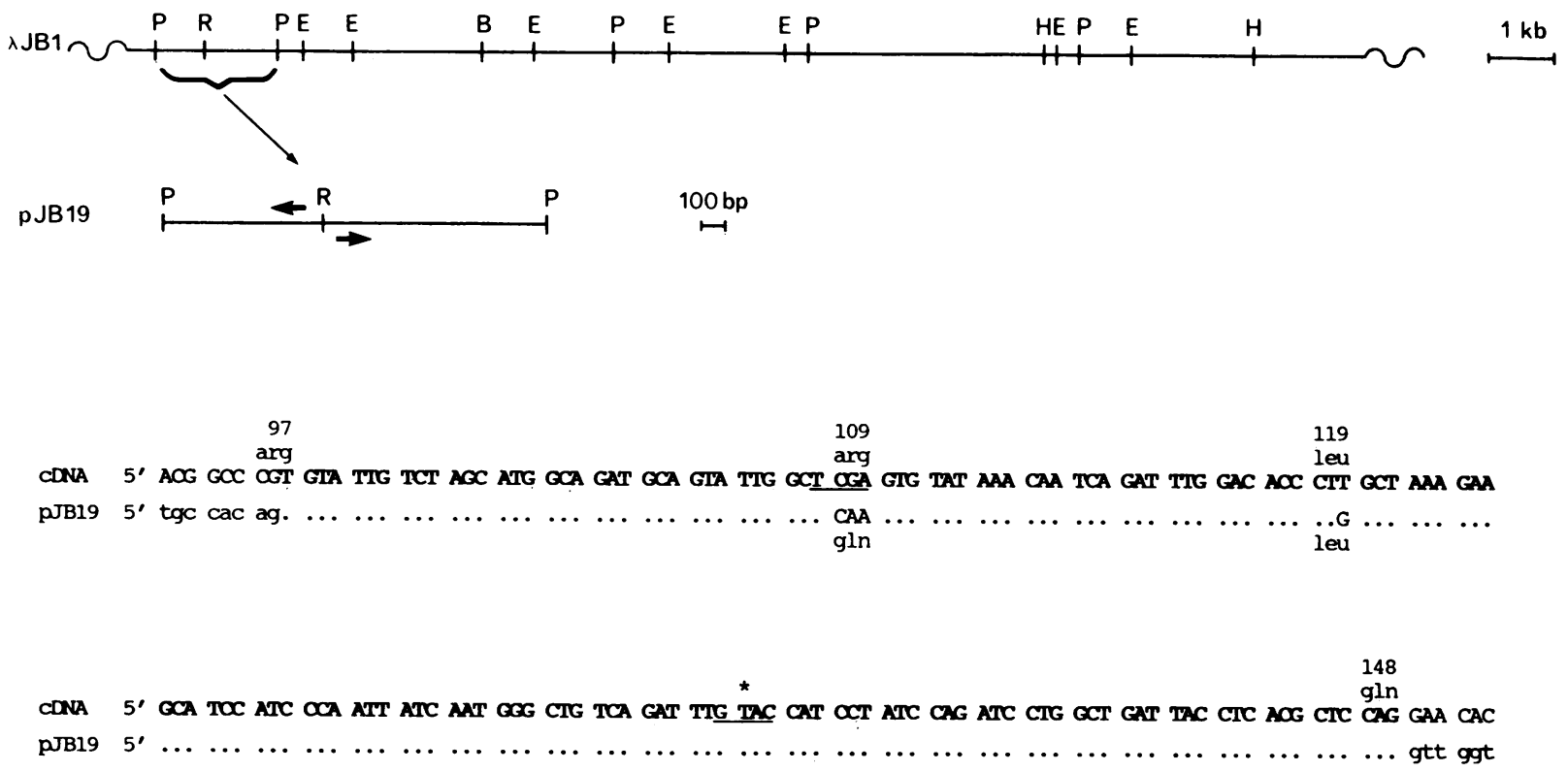

Figure 2. Isolation scheme and nucleotide sequence of a portion of the OTC gene of proband $\mathrm{Bo} . \lambda \mathrm{JB} 1$ is a phage clone containing 18 kb of DNA from the OTC gene. pJB19 is a plasmid clone containing a 1.7-kb Pst I fragment from the $5^{\prime}$ end of $\lambda J B 1$. Restriction sites for the following enzymes were located: P, Pst I; R, Rsa I; E, Eco RI; B, Bam HI; H, Hind III. The pJB19 insert was cut at the Rsa I site, subcloned into $M 13$, and sequenced as indicated by the arrows. The re- sulting nucleotide sequence is compared with the published (15) OTC cDNA sequence. Dots indicate identity; lower case letters indicate presumed intron sequences. Numbers refer to amino acid positions in the mature monomer, which has a total of 322 residues (15). An asterisk indicates the Rsa I site (GTAC). A silent base change was found at $\mathrm{Leu}_{119}$. A missense substitution was found at $\mathrm{Arg}_{109}$, which is within a Taq I site (TCGA). 
occur to a 1.7-kb Pst I fragment, for the following reason: in genomic DNA a Pst I fragment of $1.7 \mathrm{~kb}$ was known to hybridize to cDNA probe pHOC1-A, and to have an internal Taq I site in DNA from controls but not from proband Bo (data not shown). The 1.7-kb Pst I fragment seen in Southern blots corresponds to the 1.7-kb Pst I fragment in pJB19 derived from the genomic insert in $\lambda \mathrm{JB} 1$.

Autoradiographs from these experiments are shown in Fig. 3. Four identical blots were prepared from Pst I digests of the male proband of family Bo (lane 1 ), a normal male control (lane 2), the carrier mother of family En (lane 3), and the female proband of family Ma (lane 4). In panel $a$ the complete hybridization pattern of the full length cDNA is shown, including the 1.7-kb fragment which is present in all samples (arrow). The greater intensity in the female samples corresponds to the dosage of $\mathrm{X}$ chromosomes. In panel $b$, the probe was the arginine-specific oligonucleotide corresponding to the normal sequence. Signals of equal intensity are seen at $1.7 \mathrm{~kb}$ from the male control and the two heterozygous females, but not the family Bo proband. In panel $c$, the probe corresponding to the glutamine substitution in proband Bo hybridizes with his DNA in lane 1 but not with the control in lane 2, as expected. In lane 3 , hybridization with this probe indicates that the mutation found in family Bo is also present in family En. Proband Ma did not share this sequence, but in panel $d$, lane 4, her mutation was found to be the premature stop codon resulting from a $C: G$ to $T: A$ transition.

\section{Discussion}

Recurrent mutations at $C G$ dinucleotides. Three unrelated individuals with OTC deficiency who shared an anomalous Taq I restriction fragment do in fact have point mutations in the same Taq I site within an exon of the OTC gene. All three mutations are of the type proposed to occur at methylcytosines in CG dinucleotides. Given the reading frame T CGA, direct C to $T$ transition in the sense strand would create the stop codon; $\mathrm{C}$ to $\mathrm{T}$ transition in the antisense strand would, upon replication, lead to the $G$ to $A$ missense substitution. In all three instances, this X-linked mutation first appeared in the somatic tissues of a female and thus could have occurred in either male or female gametogenesis. It may be possible to trace the parental origin of these mutations by means of RFLP analysis of appropriate family members.

Structural impact of the mutations. The nonsense mutation was observed in a symptomatic female, who evidently had insufficient numbers of hepatic cells with the normal OTC gene active to prevent protein intolerance and hyperammonemia. The polypeptide produced from this allele would be truncated at less than half its normal length and completely lack the carboxyterminal residues proposed to be involved in ornithine binding (20). We have not observed this mutation in the hemizygous state, but we expect that it would confer complete OTC deficiency.

The missense substitution of glutamine for arginine 109 was observed in two male neonates. No OTC activity was measurable in a post-mortem liver sample from proband En. Although enzyme assay was not performed in proband Bo, he had a similar clinical and biochemical picture to that of proband En. The difference in lifespan probably reflects different postnatal treatment regimens rather than genetic heterogeneity. Unfortunately, studies on the quantity or biochemical properties of the OTC protein were not performed in either of these patients. Nevertheless, we believe that the substitution at codon 109 is the cause of the severe OTC deficiency in both patients. By introducing this substitution in expressed recombinant human OTC clones, it will be possible to determine its impact. $\mathrm{Arg}_{109}$ is conserved in ornithine and aspartate trans- a

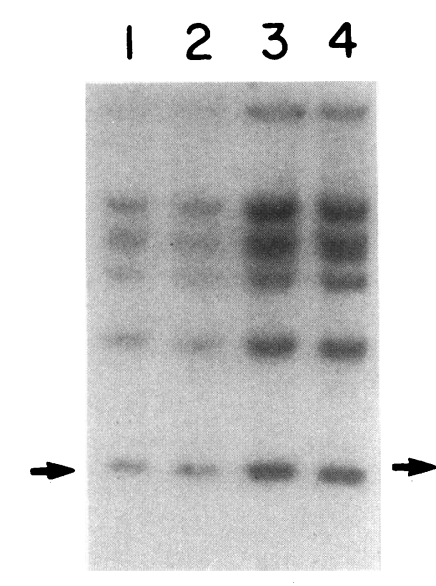

probe: b

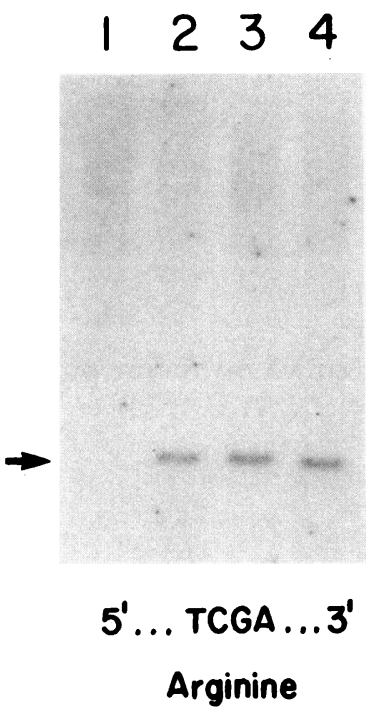

Figure 3. Hybridization of specific oligonucleotide probes to samples representing families Bo, En, and Ma. Four identical blots contain Pst I digested DNA from the male proband of family Bo (lane 1), normal male control (lane 2), carrier mother of family En (lane 3), and female proband of family $\mathrm{Ma}$ (lane 4). The probe in $a$ is the
C

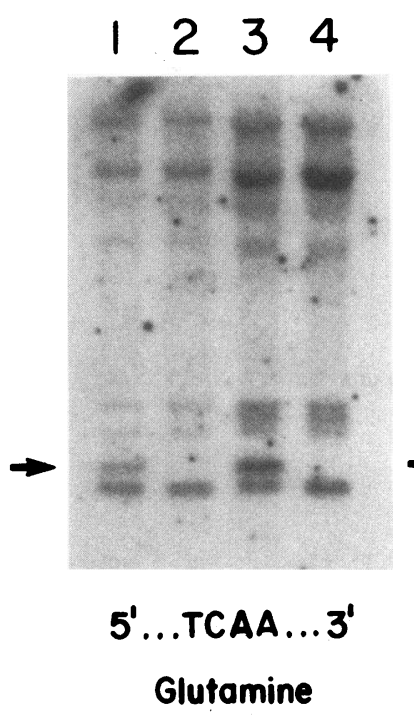

d

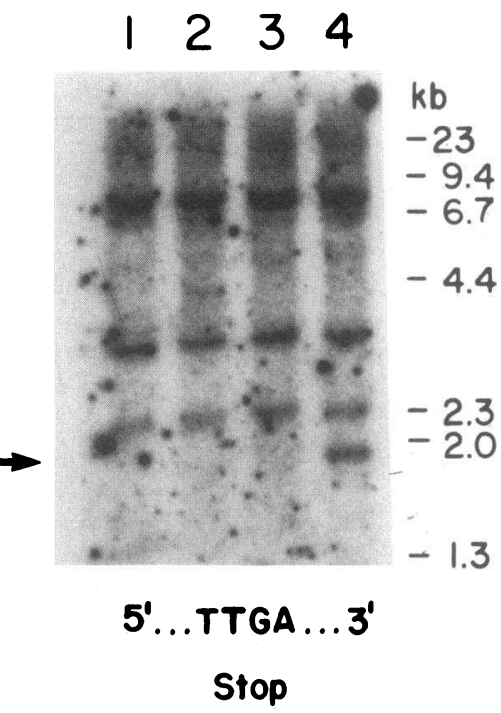

CDNA pHOC3. $b-d$ were hybridized with oligonucleotide probes specific for the sequences and codons indicated. Arrow, the position of the 1.7-kb Pst I fragment that contains the exon of interest. (N.B.: Other Pst I bands in $c$ and $d$ are artifacts of hybridization of these particular 18-mers to sequences elsewhere in the genome.) 
carbamylases from human, rat, and several microorganisms (reviewed in reference 21). It is thought to be associated with the active site, but its function has not been conclusively determined.

High risk sites. In our studies, we have shown an apparently high rate of transition mutations within a particular arginine-encoding Taq I site in the OTC gene in three patients with OTC deficiency. These findings are in accord with studies of the Factor VIII gene in hemophilia A, where seven independent mutations were shown to occur in exon sequences at four Taq 1 sites that have this reading frame. The seven cases include two nonsense mutations in exon 18 , two nonsense mutations in exon 22 , one nonsense mutation in exon 24 , and one nonsense and one missense (arginine to glutamine) in exon 26 (22-24). The exon 26 example exactly parallels our OTC cases, with the difference that in Factor VIII, the missense substitution had a distinctly milder phenotype (23).

Thus, a number of factors are involved in determining whether a $C: G$ to $T: A$ transition mutation in the internal $C G$ dinucleotide of a Taq I site will be phenotypically significant. One factor is whether the Taq I site resides in coding sequence. If so, its reading frame is another factor because if the Taq I site forms an arginine codon, such transition mutations will result in missense or nonsense mutations rather than silent substitutions. A third factor is the placement of the CGA codon in the coding region, since the effect of nonsense and missense mutations on protein function will depend on the position and role of the arginine residue.

There are three other Taq I sites in the OTC cDNA, all with the same reading frame. They are located at $\mathrm{Arg}_{-10}, \mathrm{Arg}_{60}$, and $\mathrm{Arg}_{288}$ (15). Judging from the published partial human and full rat genomic arrangements $(25,26)$, all three exist intact in genomic DNA. If indeed they are subject to methylation, then point mutations at all three sites should appear among OTC deficiencies and be easily recognized by Taq I analysis. As a case in point, the Taq I site at $\mathrm{Arg}_{288}$ is implicated as the site of a new mutation in the mother of an additional OTC-deficient boy whose Southern blot (Spence, J. E., and W. E. O'Brien, manuscript in preparation) shows a Taq I fragment length change from 2.7 to $4.4 \mathrm{~kb}$. Furthermore, at codons -10 and 60 , in addition to the obvious impact of early stop codons, missense substitutions would appear to be very detrimental.

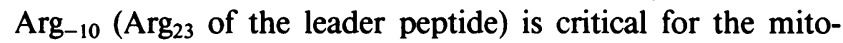
chondrial targeting and proteolytic processing of the OTC precursor (27), and Arg 60 is conserved in all carbamyltransferases (reviewed in reference 21) and is thought to be part of the carbamyl phosphate binding site (28). Naturally occurring mutations at the latter two sites have not yet been reported.

OTC deficiency remains a heterogeneous entity caused by a variety of defects in the OTC gene, the majority of which are yet undefined. Each new mutation is potentially different; nevertheless, our cases Bo and En represent the first examples of independent recurrence of identical point mutations in this gene. As missense substitutions, they will also allow inferences to be drawn about the role of $\mathrm{Arg}_{109}$ in assembly and function of the mature enzyme. Together with the nonsense mutation of case Ma, they point to a codon in the OTC gene that is both susceptible to a common mutational mechanism and located in a sensitive position. Because this codon fortuitously contributes to a Taq I site in the normal gene, mutations like these are readily detectable by simple restriction analysis.

\section{Acknowledgments}

We thank Dr. Savio Woo for the gift of a human liver cDNA library and Drs. Anthony DiLella and Jane Gitschier for helpful advice on preparing and using oligonucleotide probes.

This work was performed in the Howard Hughes Medical Institute Laboratories at the University of Pennsylvania, where R. L. Nussbaum is an Associate Investigator and A. Maddalena is an Associate, and in Hughes Institute Laboratories at Baylor College of Medicine, where W. E. O'Brien is a Senior Associate. The work was also supported in part by March of Dimes Basil O'Connor grant 5-444 to R. L. Nussbaum.

\section{References}

1. Walser, M. 1983. Urea cycle disorders and other hyperammonemic syndromes. In The Metabolic Basis of Inherited Disease. J. Stanbury, J. Wyngaarden, D. Fredrickson, J. Goldstein, and M. Brown, editors. McGraw-Hill Book Co., Inc., New York. 402-438.

2. Briand, P., B. Francois, D. Rabier, and L. Cathelineau. 1982. Ornithine transcarbamylase deficiencies in human males: kinetic and immunochemical classification. Biochim. Biophys. Acta. 704:100106.

3. Saheki, T., Y. Imamura, I. Inoue, S. Miura, M. Mori, A. Ohtake, M. Tatibana, N. Katsumata, and T. Ohno. 1984. Molecular basis of ornithine transcarbamylase deficiency lacking enzyme protein. J. Inherited Metab. Dis. 7:2-8.

4. Rozen, R., J. E. Fox, W. A. Fenton, A. L. Horwich, and L. E. Rosenberg. 1985. Gene deletion and restriction fragment length polymorphisms at the human ornithine transcarbamylase locus. Nature (Lond.). 313:815-817.

5. Old, J. M., P. L. Briand, S. Purvis-Smith, N. J. Howard, B. Wilcken, J. Hammond, P. Pearson, L. Cathelineau, R. Williamson, and K. E. Davies. 1985. Prenatal exclusion of ornithine transcarbamylase deficiency by direct gene analysis. Lancet. i:73-75.

6. Rozen, R., J. E. Fox, A. M. Hack, W. A. Fenton, A. L. Horwich, and L. E. Rosenberg. 1986. DNA analysis for ornithine transcarbamylase deficiency. J. Inherited Metab. Dis. 9(Suppl. 1):49-57.

7. McClead, R. E., Jr., R. Rozen, J. Fox, L. Rosenberg, J. Menke, R. Bickers, and G. Morrow III. 1986. Clinical application of DNA analysis in a family with OTC deficiency. Am. J. Med. Genet. 25:513518.

8. Rosenberg, L. E., F. Kalousek, and M. D. Orsulak. 1983. Biogenesis of ornithine transcarbamylase in $s p f^{\text {ash }}$ mutant mice: two cytoplasmic precursors, one mitochondrial enzyme. Science (Wash. DC). 222:426-428.

9. Veres, G., R. A. Gibbs, S. E. Scherer, C. T. Caskey. 1987. The molecular basis of the sparse fur mouse mutation. Science (Wash. DC). 237:415-417.

10. Pembrey, M., J. Old, J. Leonard, C. Rodeck, R. Warren, and K. Davies. 1985. Prenatal diagnosis of ornithine carbamoyl transferase deficiency using a gene specific probe. J. Med. Genet. 22:462-465.

11. Fox, J., A. Hack, W. Fenton, M. Golbus, S. Winter, F. Kalousek, R. Rozen, S. Brusilow, and L. Rosenberg. 1986. Prenatal diagnosis of ornithine transcarbamylase deficiency with use of DNA polymorphisms. N. Engl. J. Med. 315:1205-1208.

12. Fox, J. E., A. M. Hack, W. A. Fenton, and L. E. Rosenberg. 1986. Identification and application of additional restriction fragment length polymorphisms at the human ornithine transcarbamylase locus. Am. J. Hum. Genet. 38:841-847.

13. Barker, D., M. Shafer, and R. White. 1984. Restriction sites containing CpG show a higher frequency of polymorphism in human DNA. Cell. 36:131-138.

14. Nussbaum, R., B. Boggs, A. Beaudet, S. Doyle, J. Potter, and W. O'Brien. 1986. New mutation and prenatal diagnosis in ornithine transcarbamylase deficiency. Am. J. Hum. Genet. 38:149-158. 
15. Feinberg, A. P., and B. Vogelstein. 1984. A technique for radiolabeling DNA restriction endonuclease fragments to high specific activity. Anal. Biochem. 132:6-13. Addendum, 137:266-267.

16. Studencki, A., and B. Wallace. 1984. Allele-specific hybridization using oligonucleotide probes of very high specific activity: discrimination of the human beta-A and beta-S globin genes. DNA (NY). 3:7-15.

17. Wood, W., J. Gitschier, L. Lasky, and R. Lawn. 1985. Base composition-independent hybridization in tetramethylammonium chloride: a method for oligonucleotide screening of highly complex gene libraries. Proc. Natl. Acad. Sci. USA. 82:1585-1588.

18. DiLella, A., and S. L. C. Woo. 1987. Hybridization of genomic DNA to oligonucleotide probes in the presence of tetramethylammonium chloride. Methods Enzymol. 152:447-450.

19. Horwich, A., W. Fenton, K. Williams, F. Kalousek, J. Kraus, R. Doolittle, W. Konigsberg, and L. Rosenberg. 1984. Structure and expression of a complementary DNA for the nuclear coded precursor of human mitochondrial ornithine transcarbamylase. Science (Wash. DC). 224:1068-1074.

20. Marshall, M., and P. Cohen. 1980. Ornithine transcarbamylases. Ordering of S-cyano peptides and location of characteristically reactive cysteinyl residues within the sequence. J. Biol. Chem. 255:7287-7290.

21. Huygen, R., M. Crabeel, and N. Glandsdorff. 1987. Nucleotide sequence of the $A R G 3$ gene of the yeast Saccharomyces cerevisiae encoding ornithine carbamoyltransferase: comparison with other carbamoyltransferases. Eur. J. Biochem. 166:371-377.
22. Gitschier, J., W. Wood, E. Tuddenham, M. Schuman, T. Goralka, E. Chen, and R. Lawn. 1985. Detection and sequence of mutations in the factor VIII gene of haemophiliacs. Nature (Lond.). 315:427-430.

23. Gitschier, J., W. Wood, M. Schuman, and R. Lawn. 1986. Identification of a missense mutation in the Factor VIII gene of a mild hemophiliac. Science (Wash. DC). 232:1415-1416.

24. Youssoufian, H., H. Kazazian, D. Phillips, S. Aronis, G. Tsiftis, V. Brown, and S. Antonarakis. 1986. Recurrent mutations in haemophilia A give evidence for CpG mutation hotspots. Nature (Lond.). 324:380-382.

25. Hata, A., T. Tsuzuki, K. Shimada, M. Takiguchi, M. Mori, and I. Matsuda. 1986. Isolation and characterization of the human ornithine transcarbamylase gene: structure of the 5'-end region. J. Biochem. (Tokyo). 100:717-725.

26. Takiguchi, M., T. Murakami, S. Miura, and M. Mori. 1987. Structure of the rat ornithine transcarbamylase gene, a large $\mathrm{X}$ chromosome-linked gene with an atypical promoter. Proc. Natl. Acad. Sci. USA. 84:6136-6140.

27. Horwich, A., F. Kalousek, W. Fenton, R. Pollock, and L. Rosenberg. 1986. Targeting of pre-ornithine transcarbamylase to mitochondria: definition of critical regions and residues in the leader peptide. Cell. 44:451-459.

28. Marshall, M., and P. Cohen. 1980. Evidence for an exceptionally reactive arginyl residue at the binding site for carbamyl phosphate in bovine ornithine transferase. J. Biol. Chem. 255:7301-7305. 